INPLASY

PROTOCOL

To cite: Wang et al. Mortality risk with insulin use in patients with COVID-19 and diabetes:

A meta-analysis. Inplasy protocol 202110052. doi:

10.37766/inplasy2021.1.0052

Received: 14 January 2021

Published: 15 January 2021

Corresponding author:

WT Wang

wt19960219@163.com

Author Affiliation:

The Affiliated Hospital of

Qingdao University

Support: No Support.

Review Stage at time of this submission: Preliminary searches.

Conflicts of interest:

None.

\section{Mortality risk with insulin use in patients with COVID-19 and diabetes: A meta-analysis}

\author{
Wang, WT1; Sun, Y2; Sun, YB³.
}

Review question / Objective: The study includs patients with confirmed COVID-19 and diabetes, with data available to compare the risk of mortality among insulin users compared to non-insulin users. And exclusion criteria includes review, basic medical experiments, case reports, letters and other non-clinical studies; Incomplete data; Small number of sample and low quality literature.Our study have no intervention because it is an observational study. Outcome indicators for the study were death in diabetes and COVID-19 patients. We're going to compare mortality rates in insulin group and noinsulin group.

Condition being studied: The outbreak of COVID-19 at the end of 2019 has brought great challenges to the world's medical health in the past year. It has been reported that COVID-19 patients with diabetes have a high mortality rate, and wellcontrolled blood glucose is related to lower mortality. Insulin is a commonly used hypoglycemic drug in clinical practice, so we discuss the influence of insulin on mortality of such patients.

INPLASY registration number: This protocol was registered with the International Platform of Registered Systematic Review and Meta-Analysis Protocols (INPLASY) on 15 January 2021 and was last updated on 15 January 2021 (registration number INPLASY202110052).

\section{INTRODUCTION}

Review question / Objective: The study includs patients with confirmed COVID-19 and diabetes, with data available to compare the risk of mortality among insulin users compared to non-insulin users. And exclusion criteria includes review, basic medical experiments, case reports, letters and other non-clinical studies; Incomplete data; Small number of sample and low quality literature. Our study have no intervention because it is an observational study. Outcome indicators for the study 
were death in diabetes and COVID-19 patients. We're going to compare mortality rates in insulin group and noinsulin group.

Condition being studied: The outbreak of COVID-19 at the end of 2019 has brought great challenges to the world's medical health in the past year. It has been reported that COVID-19 patients with diabetes have a high mortality rate, and well-controlled blood glucose is related to lower mortality. Insulin is a commonly used hypoglycemic drug in clinical practice, so we discuss the influence of insulin on mortality of such patients.

\section{METHODS}

Participant or population: three reviewers will apply Inclusion criteria and select studies for inclusion in the systematic review, one will screen and an other check decisions, and If there is some individual judgements it will be resolved by discussion or by a third reviewers.

Intervention: No intervention.

Comparator: We're going to compare mortality rates in insulin group and noinsulin group.

Study designs to be included: The included study are of cohort or case-control design.

Eligibility criteria: Inclusion criteria: the study are of cohort or case-control design, included patients with confirmed COVID-19 and diabetes, with data available to compare the risk of mortality among insulin users compared to non-insulin users. Exclusion criteria: review, basic medical experiments, case reports, letters and other non-clinical studies; Incomplete data;Small number of sample and low quality literature.

Information sources: We will retrieve the Embase, MEDLINE, PubMed, the Cochrane Library databases, To extract information on diabetes and COVID-19 until 2020.12.20.
Main outcome(s): Outcome indicators for the study are the death in diabetes and COVID-19 patients.

Quality assessment / Risk of bias analysis: We will evaluate the quality of the included articles through Newcastle-Ottawa Scale.

Strategy of data synthesis: The key words included "2019 novel coronavirus disease", "COVID-19", "coronavirus disease 2019", "SARS-cov-2", "diabetes mellitus", "glycuresis", "diabetic", "Insulin", and "lletin".

Subgroup analysis: We will conduct subrent analysis through sensitivity analysis and heterogeneous results, such as age, research methods, etc.

Sensibility analysis: We will conduct sensitivity analysis by excluding one study at a time, and recalculating the remaining studies.

Country(ies) involved: China.

Keywords: Mortality COVID-19 diabetes insulin. 